\title{
Comprehensive Nuclear-Test-Ban Treaty - a peace-keeping initiative with scientific impact
}

\author{
Tine B. Larsen, Peter H. Voss, Trine Dahl-Jensen and Søren Gregersen
}

Any major shaking of the Earth can be recorded on a seismograph regardless of the nature of the source. Earthquakes and large explosions generate waves with similar frequency content. This fact has been used for decades to construct systems to monitor detonations of underground nuclear explosions. The quality of the monitoring system has increased significantly in recent years, and we demonstrate here that the data are useful in Danish earthquake research.

One important difference between explosions and earthquakes is the depth of the source, most earthquakes occurring at much larger depths than explosions. Thus the depth determination is important in both fields. However, accurate depth determination of earthquake hypocentres is a challenge even when it comes to large well-recorded earthquakes. The uncertainty of the calculated depth of a Danish earthquake is of the same magnitude as the depth itself when using standard location techniques. A technique utilising crustal phases recorded at large distances has been introduced at the Geological Survey of Denmark and Greenland to improve the determination of the hypocentre depths.

Only the largest earthquakes in Denmark and its immediate surroundings produce sufficiently strong signals to be recorded at teleseismic distances, i.e. larger than $3000 \mathrm{~km}$. The signals are discernable at low-noise seismic array sta-

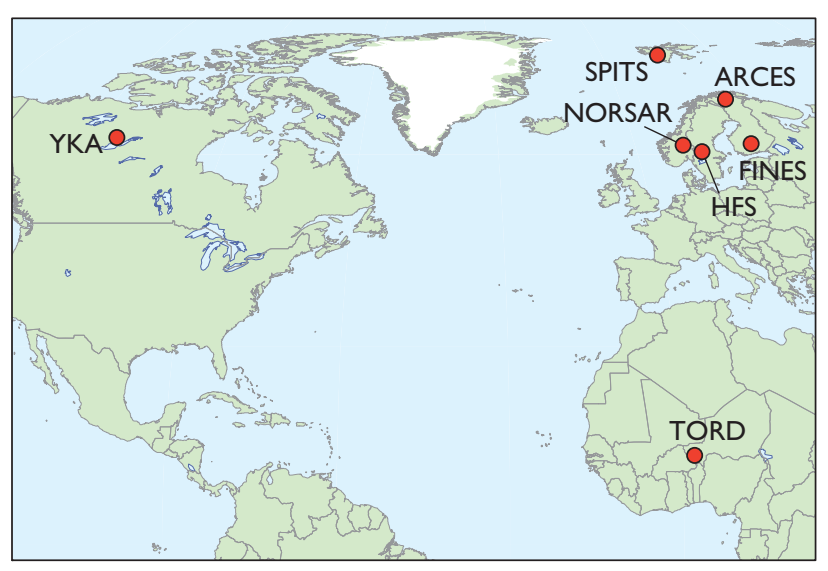

Fig. 1. Map of the North Atlantic region showing the locations of seismic array stations used by GEUS to locate earthquakes in Denmark and Greenland. YKA, Canada. SPITS, NORSAR and ARCES, Norway. FINES, Finland. HFS, Sweden. TORD, Niger. tions as far away as North America and Africa. Some of these stations are operated by the United Nations Comprehensive Nuclear-Test-Ban Treaty Organisation (CTBTO). Data from many of these stations are available for scientific purposes. We demonstrate here how data from Canada and Niger can significantly improve the depth estimates for two earthquakes, one in Skåne, Sweden in 2008 and the other in the Danish part of the North Sea in 2010 (Figs 1, 2).

\section{CTBTO and IMS}

The Comprehensive Nuclear-Test-Ban Treaty was adopted by the general assembly of the United Nations (UN) in September 1996. The treaty bans all nuclear explosions on Earth whether for military or for peaceful purposes. Denmark signed the treaty in 1996, and it was ratified by the Parliament of Denmark in 1998. The treaty will enter into force once it has been signed and ratified by all nuclear powers of the world. However, the ratification is still pending in several key countries such as China, Pakistan, India and United States of America.

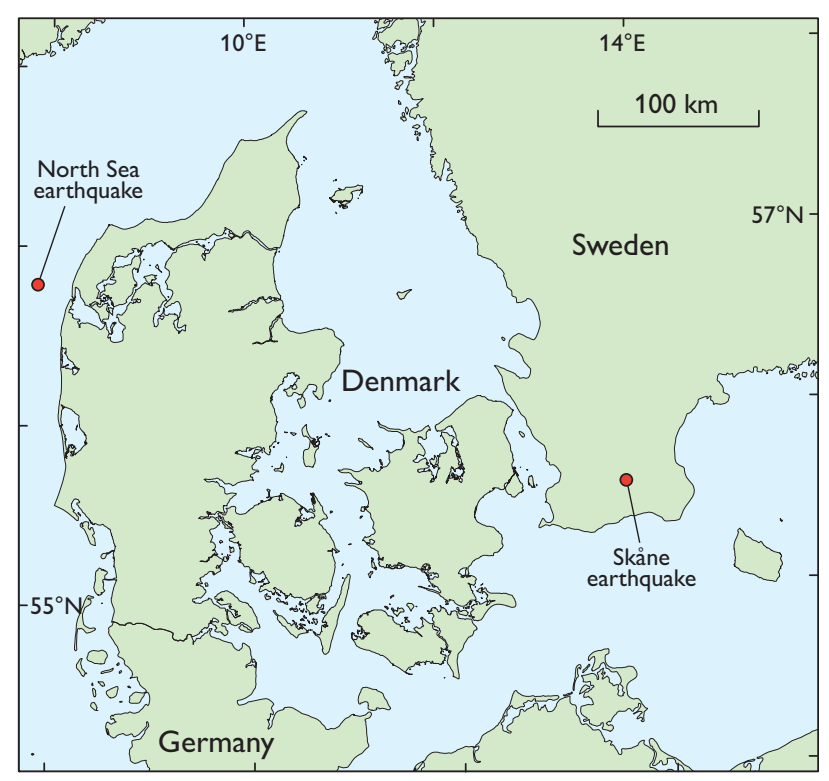

Fig. 2. Teleseismic array data have been used to locate earthquakes and measure their depths in Skåne on 16 December 2008 and in the North Sea on 19 February 2010. 
Significant resources are being allocated to the development of a large international monitoring system (IMS), so that all technical systems as well as procedures, manuals and other agreements are in place once the treaty enters into force. The IMS is a UN-controlled monitoring system based on four technologies: seismology, radio nuclides, hydroacoustics and infrasound. The system consists of a worldwide network of high-quality monitoring stations, supplementing the existing national networks of detectors, and it has improved the detection threshold for explosions as well as earthquakes in large parts of the Earth. Data from the stations are transmitted via secure satellite links to a UN data centre in Vienna.

All the data are processed at the centre in Vienna. The data centre is completely neutral and is not permitted to judge if an event is natural, such as an earthquake or a volcanic eruption, or caused by a man-made explosion. Instead the data centre makes raw data as well as processed data available to the individual countries, and then it is up to the national authority in each country to decide whether an event is suspicious or not.

Should an event be deemed suspicious by a country, any country has the right to request further processing by the IMS and ultimately request an on-site inspection at the location of the suspicious event. In recent years, nuclear test explosions have been easy to identify, as the involved nations have openly announced the tests and provided information about location and time of the explosions (Pakistan 1998, India 1998, North Korea 2006 and 2009).

\section{GEUS and CTBTO}

In Denmark, GEUS houses both the national data centre and is also the National Authority for the CTBTO. GEUS is responsible for running two monitoring stations in the IMS network. One is a seismograph in Kangerlussuaq, West Greenland; it is part of the auxiliary (secondary) seismic network. The other is an infrasound station near Qaanaaq, North-West Greenland, that is a primary IMS station. The Danish Meteorological Institute takes part in the daily maintenance of the infrasound station.

Together with diplomats from the Danish embassy in Vienna, GEUS is also involved in the preparatory work on operational manuals and procedures for waveform processing that is carried out at the UN centre. This involves discussions on how to tune the detection system to automatically filter out as many earthquake signals as possible, so that the system triggers on explosions only. This part of the system still needs significant improvements.
In order to be as familiar as possible with the data from the IMS before the treaty enters into force, seismologists at GEUS make experiments with the incoming data for other purposes. Recently we have found that the IMS raw seismological data are useful for determining accurate depths of Danish earthquakes. The IMS seismographs are of a very high quality and it is possible to identify signals from relatively weak earthquakes at distant stations.

\section{Earthquake depth analysed using CTBTO array data}

For several decades we have supplemented with data from seismic stations in the countries around Denmark and Greenland to help detect and localise earthquakes. Seismic array stations produce data of particularly high quality. A seismic array station consists of a large number of sensors installed in a small area. The ability to detect an earthquake decreases when the strength of the seismic noise increases relative to the strength of the seismic signal. Seismic noise can be reduced significantly in earthquake data recorded by an array by summing the signals recorded at the sensors, thus enabling the detection of smaller events than is possible on a station with just one sensor. The closest seismic arrays that contribute to the monitoring of earthquakes in Denmark and Greenland are the NORSAR and ARCES arrays in Norway, the HFS array in Sweden, the FINES array in Finland and the SPITS array at Svalbard (Fig. 1).

Previously, array data have been used to locate earthquakes in Denmark and Greenland from observations of $P$ and $S$ phase travel times only. For the two earthquakes in Skåne on 16 December 2008 and in the North Sea on 19 February 2010, however, a new technique was used to improve the estimate of the depth of the earthquakes. This

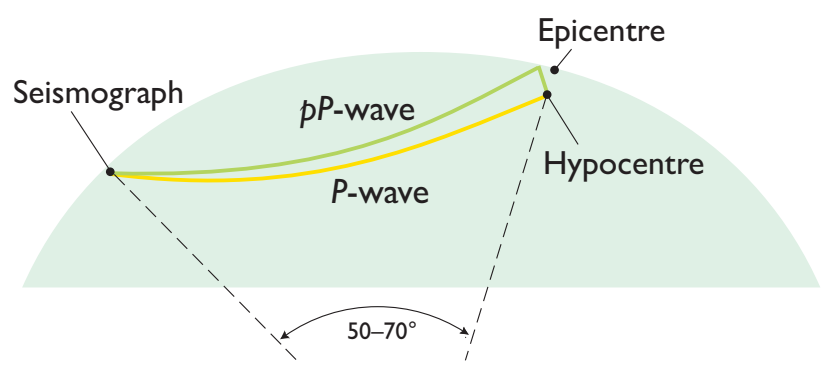

Fig. 3. The slightly different paths taken by the $P$ - and the $p P$ - (or $s P$-) waves can be used to calculate the depth of an earthquake. The $P$-wave (yellow) travels directly from the hypocentre to the seismograph, whereas $p P$ - and $s P$-waves (green) travel from the hypocentre to the surface near the epicentre and from there reflected to the seismograph. The paths of the $p P$ - and $s P$-waves differ slightly due to the crustal structures near the earthquake. 
technique uses teleseismic observations of travel-time differences between the $P$ phase and the $p P$ and/or $s P$ phases to calculate the depth of the earthquake. The $p P$ and $s P$ phases are reflections at the Earth's surface of the shaking from the earthquake hypocentre. These phases are recorded slightly after the $P$ wave arrival (Fig. 3). The technique requires measurements with a high signal-to-noise ratio and good knowledge of the geological structures near the epicentre. The principles of the teleseismic depth-determination technique are very simple. At teleseismic distances the difference in travel length is negligible for the $P$-wave travelling directly from the hypocentre to the seismograph and the part of the $p P$-wave travelling from the epicentre to the seismograph (Fig. 3). The difference in travel time between the phases is assumed to be caused by the $p P$-wave travelling almost vertically through the crust from the hypocentre to the surface. If the velocity structure in the crust below the epicentre is well known, the travel time difference between $P$ and $p P$ and/or $s P$ can be converted to a depth. An accurate crustal velocity model is therefore critical for the analysis since errors in the crustal model will give a wrong determination of the depth. A teleseismic recording is needed as the paths of the $p P$ and $s P$ phases must be near vertical at the source. However, the distance between the earthquake and the seismic array must be sufficiently short for the phase not to be effected by the core-mantle boundary. We find that a distance of around 50 to 70 degrees is optimal for this technique.

A significant number of the IMS stations are seismic arrays. The stations are installed at locations with low ambient noise, in order to record data of high quality. In our analysis we use data from the Yellowknife Array (YKA) in Canada and the Toridu Array (TORD) in Niger (Fig. 1). The Yellowknife Array consists of 19 short-period sensors and 4 broadband sensors. The sensors are installed in a cross with an aperture of $25 \mathrm{~km}$. The array was installed in 1962 with the main purpose of monitoring underground nuclear explosions (source: Natural Resources Canada). The Toridu Array is a modern array constructed specifically for the CTBTO (Estabrook et al. 2009). It consists of 16 broadband sensors deployed in three concentric rings with a central node. The sensors are not radially aligned, as this layout leads to the largest noise reduction (e.g., Schweitzer et al. 2002).

As described above, the energy release of the majority of the earthquakes in Denmark and Greenland is too low to generate clear signals even at the best IMS stations. The two earthquakes, Skåne, 16 December 2008 and the North Sea, 19 February 2010, measuring 4.8 and 4.7 on the Richter scale, respectively, are our best candidates for this technique (Fig. 2). The geological structures are well mapped in Denmark where we have a good knowledge of $P$-wave velocity
(Thybo 2001). For the $S$-wave velocity we have used $V p=$ $1.73 \times V s$ to estimate the earthquake depth. Using this technique in Greenland will be less reliable in most areas, because the crustal structures are not as well mapped.

We have analysed the measurements of the Skåne earthquake on the YKA Array (Fig. 4). The measurements show a good signal-to-noise ratio and we find a difference in the $P$ and $s P$ travel times of $c .4 \mathrm{sec}$., equivalent to an earthquake depth of $9 \mathrm{~km}$. The previously calculated depth of this earthquake was $18.1 \pm 5.2 \mathrm{~km}$ using the standard location method (SNSN 2010), and the result from moment-tensor inversion was $8 \mathrm{~km}$ (Regel 2010). The standard location method is based on an approach that searches for the hypocentre that gives the best fit to measured travel times of $P$ - and $S$-waves within $c .1000 \mathrm{~km}$ of the earthquake, using a 1D Earth model. The larger depth obtained by SNSN might be due to the velocity model, which is not well calibrated for the Skåne

A Plot start time:

B

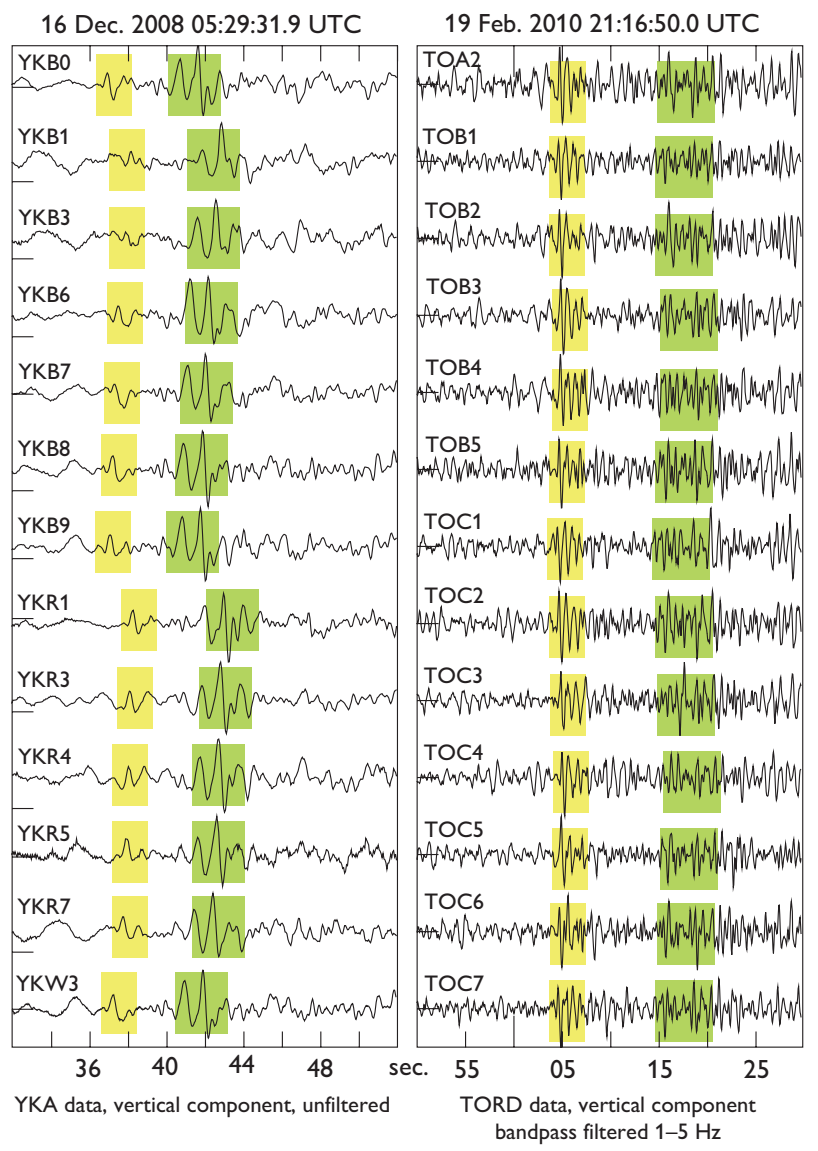

Fig 4. A: The $P$-wave train from the Skåne earthquake on the Yellowknife Array (YKA). Yellow is the direct $P$ phase and green is the $s P$ phase. B: The $P$-wave train from the North Sea earthquake on the Toridu Array (TORD). Yellow is the direct $P$ phase and green is the $p P$ phase. The high number of sensors improves the possibility of identifying different phases. 
area (B. Lund, personal communication 2010) and the use of measurements far from the epicentre.

The North Sea earthquake measurements on the YKA have a low signal-to-noise ratio on many of the sensors, but on the best five sensors we observe a signal $c .15 \mathrm{sec}$. after the $P$ phase. Interpreting this as the $s P$ phase yields a depth of $35.2 \mathrm{~km}$. The high noise level in the YKA data carries a risk of misinterpretation of the data. We therefore supplement with data from the TORD array (Fig. 4), to verify the depth of this earthquake. From the measurements of the TORD array we find a difference in the $P$ and $p P$ travel time of $c .11$ sec., corresponding to an earthquake depth of $38.5 \mathrm{~km}$. The previously calculated depth was $38.7 \pm 10.3 \mathrm{~km}$ using the standard location method. The depth uncertainty is larger than that of the Skåne earthquake due the larger distance of the nearest seismometer. The depths of the two earthquakes are comparable with the depths of previous earthquakes in these areas (Gregersen et al. 1999). The higher noise level of the North Sea event (Fig. 4) is a source of error in the analysis. The higher noise level could be due to an energy radiation pattern of the earthquake that is low in the direction of TORD or a different frequency content of the released shaking.

\section{Concluding remarks}

Teleseismic array data have produced consistent depth estimates for two recent earthquakes in the Danish area. This raises the possibility that we might find other earthquakes suitable for this technique in the GEUS database, especially from Greenland.

The seismological involvement in detection and discrimination of nuclear explosions has spurred significant Nordic collaboration since the 1960s, and a yearly Nordic seismological meeting is held. GEUS has made a special contribution together with the UK Foreign and Commonwealth Office, NORSAR, the Swedish National Defence Research Establishment and the University of Helsinki to improve data exchange between the International Monitoring System and the International Seismological Centre. This has resulted in the development of a collection of interactive seismological tools for merging and manipulating the two largest and most complete seismological databases (Gaspa et al. 2010). In the future this will hopefully help improve and ease the scientific use of the International Monitoring System data.

\section{References}

Comprehensive Nuclear Test-Ban Treaty (www.ctbto.org).

Estabrook, C., Bergsson, B., Soumana, S., Boureima, O. \& Moumouni, M. 2009: Results from IMS Seismic Array in Niger. Poster presented at the EGU meeting in Vienna, April 2009.

Gaspa, O., Bondar, I., Harris, J. \& Storchak, D. 2010: The CTBTO link to the ISC Database. The 41st Nordic Seminar on Detection Seismology, Århus, 6-8 October, 2010. Program with abstracts, 14 only.

Gregersen, S., Hjelme, J. \& Hjortenberg, E. 1998: Earthquakes in Denrnark. Bulletin of the Geological Society of Denmark 44, 115-127.

Regel, J. 2010: Moment tensor of the 16 Dec[ember] 2008 earthquake in Skåne, Sweden. The 41st Nordic Seminar on Detection Seismology, Århus, 6-8 October 2010. Program with abstracts, 39 only.

Schweitzer, J., Fyen, J., Mykkeltveit, S. \& T. Kværna, T. 2010: Seismic Arrays. In: New Manual of Seismological Observatory Practice, Chapter 9. Doi: 10.2312/GFZ.NMSOP_rl_ch9.

SNSN 2010: Swedish National Seismic Network (http://snsn.geofys. uu.se/).

Thybo, H. 2001: Crustal structure along the EGT profile across the Tornquist Fan interpreted from seismic, gravity and magnetic data. Tectonophysics 334, 155-190. 